included. Patients with amyloidosis and pregnancy were excluded. In order to evaluate the disease status, acute phase reactants and patient-reported disease severity visual analog scale (VAS) scores were analyzed. Acute phase reactants were evaluated during attack-free periods. The VAS score was reported on a scale of 0-10, 10 meaning the disease at its most severe form, and 0 meaning the least.

Results: Fifty-two patients (21 male, 31 female) with the mean age of $35.88 \pm 12.4$ years, were included in this study. The presenting signs and symptoms of the patients are shown in Figure 1. The mean age of initial symptoms and diagnosis were $12.84 \pm 10.06$ and $20.39 \pm 12.35$ years in respective order. The treatment information of the patients before and during Canakinumab injections was shown in Table 1. The mean Erythrocyte Sedimentation Rate (ESR) decreased from $25.31 \pm 20.64$ to $11.52 \pm 9.78 \mathrm{~mm} /$ hour. The mean C-reactive Protein (CRP) decreased from $28.18 \pm 47.04$ to $2.02 \pm 2.31 \mathrm{mg} / \mathrm{L}$ (both $\mathrm{p}<0.0001$ ). The mean VAS score decreased from $8.04 \pm 1.9$ to $1.4 \pm 1.73$ ( $p<0.0001$ ). Canakinumab treatment was terminated in 33 patients, 22 of which was due to successful remission. The termination of the treatment was because of pregnancy or will of pregnancy in 4 patients, inadequate treatment response 3 patients, treatment noncompliance in 2 patients, chronic hepatitis $C$ related cirrhosis in 1 patient, and change to a different biologic agent in 1 patient. The only side effect experienced was hallucinations in one patient who was already under remission.

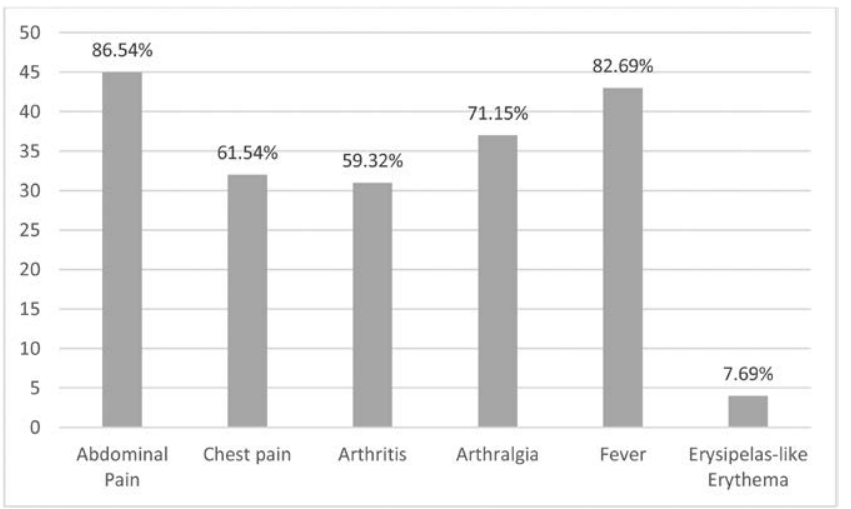

Figure 1. The Presenting Signs and Symptoms of the Patients

Table 1. The Treatment Information of the Patients

\begin{tabular}{lc}
\hline Initial Mean Daily Colchicine Dose, mg (mean \pm standard deviation) & $1.68 \pm 0.46$ \\
Mean Daily Colchicine Dose before Canakinumab Treatment, mg & $1.63 \pm 0.6$ \\
The Mean Injection Number, n & $17.5 \pm 17.8$ \\
Canakinumab Treatment Indication, n (\%) & \\
Inadequate Response to Previous Treatment & $38(73.08)$ \\
Side Effect to Previous Treatment & $8(15.38)$ \\
Poly Arteritis Nodosa & $2(3.85)$ \\
Recurrent Pericarditis & $1(1.92)$ \\
CNS Vasculitis & $2(3.85)$ \\
Poor Anakinra Treatment Adherence & $1(1.92)$ \\
FMF Encephalopathy & $1(1.92)$ \\
\hline
\end{tabular}

Conclusion: Canakinumab seems effective in controlling the subclinical inflammation and raising the quality of life of the patient. It has a favorable side effect profile. According to our single-center, real-life data, Canakinumab can be used as an alternative treatment method in colchicine resistant patients.

Disclosure of Interests: None declared

DOI: 10.1136/annrheumdis-2020-eular.5329

\section{SAT0519 DRUG RETENTION RATES OF BIOLOGICAL AGENTS IN ADULT ONSET STILL'S DISEASE IN THE PRE- CANAKINUMAB ERA}

N. Farina ${ }^{1}$, A. Tomelleri ${ }^{1}$, C. Campochiaro ${ }^{1}$, G. De Luca ${ }^{1}$, G. Cavalli ${ }^{1}$, E. Baldissera ${ }^{1}$, L. Dagna ${ }^{1} .{ }^{1}$ IRCCS San Raffaele Hospital, Unit of Immunology, Rheumatology, Allergy and Rare Diseases, Milan, Italy

Background: Adult onset Still's disease (AOSD) is a rare systemic inflammatory disease. Glucocorticoids represents first-line therapy and conventional synthetic disease-modifying anti-rheumatic drugs (csDMARDs) play a central role as steroid-sparing agents. Patients refractory to CSDMARD may require the introduction of a biological DMARD ( $b$ DMARD). There is paucity of data about the efficacy and drug retention rate (DRR) of bDMARDs used in the treatment of AOSD.

Objectives: To retrospectively evaluate DRR and reasons for discontinuation of five different bDMARDs in a monocentric cohort of AOSD patients in a real-world setting.
Methods: AOSD patients followed at our Center who received at least one bDMARD were selected. Data about disease duration, number of bDMARDs, reasons for bDMARDs discontinuation, concomitant csDMARDs were collected Survival curves were determined by the Kaplan-Meier method and compared using a stratified log-rank test. 24-month DRRs were then calculated. Hazard ratio $(\mathrm{HR})$ for previous bDMARDs was also evaluated

Results: We identified 42 patients and 79 bDMARD-courses (Table 1). Anakinra (ANK, $n=41$ ) was the most used bDMARD, followed by tocilizumab (TCZ $\mathrm{n}=21$ ) and Tumor Necrosis Factor inhibitors (TNFi, $\mathrm{n}=17$ ). At bDMARDs initiation, all patients were being given prednisone (mean dose, $23 \pm 18 \mathrm{mg} /$ day) and $76 \%$ were on concomitant csDMARD therapy. 36 (46\%) treatment courses were stopped by 24 months; discontinuation causes are shown in Table 2. 14 treatment courses were started in the previous 24 months $(A N K=9, T C Z=5)$, without being stopped before the study time but not reaching 2-year treatment duration. Overall retention rates at 24 months were the following: TCZ, 62.5\%; ANK, 53.1\%; TNFi, $11.8 \%$ ( $p=0.021)$. Survival curves are shown in Figure 1 DRRs of ANK and TCZ were statistically comparable $(p=0.576)$, and they were both significantly higher than the DRR of TNFi $(p=0.015)$. Previous bDMARDs therapy showed no effect on DRR (HR 0.73, 95\% Cl $=0.40-1.31, \mathrm{p}=0.288$ ).

Table 1. Clinical characteristics at initiation of biologic agents in adult onset Still's Disease. TNFi = Tumor Necrosis Factor inhibitors

\begin{tabular}{lcccc}
\hline & $\begin{array}{c}\text { Anakinra } \\
(\mathbf{n = 4 1 )}\end{array}$ & $\begin{array}{c}\text { Tocilizumab } \\
(\mathbf{n = 2 1 )}\end{array}$ & TNFi (n=17) & $\begin{array}{c}\mathbf{p} \\
\text { - value }\end{array}$ \\
\hline Age (years) & $38 \pm 16$ & $43 \pm 19$ & $38 \pm 13$ & 0.601 \\
Female sex (\%) & $61 \%$ & $48 \%$ & $35 \%$ & 0.443 \\
Mean disease duration (months) & $49 \pm 60$ & $72 \pm 75$ & $61 \pm 64$ & 0.445 \\
Systemic disease (\%) & $61 \%$ & $38 \%$ & $29 \%$ & 0.213 \\
C-reactive protein (mg/l) & $99 \pm 84$ & $111 \pm 100$ & $66 \pm 52$ & 0.445 \\
Erythrocyte sedimentation rate & $71 \pm 32$ & $65 \pm 27$ & $77 \pm 41$ & 0.617 \\
$\quad(m m / h)$ & & & & \\
White blood cells (cells/ul) & $14640 \pm 4540$ & $14116 \pm 3724$ & $16870 \pm 5504$ & 0.361 \\
Ferritin (ng/ml) & $10114 \pm 18657$ & $6327 \pm 8061$ & $6295 \pm 9573$ & 0.591 \\
Previous therapy (\%) & $100 \%$ & $100 \%$ & $100 \%$ & 1.000 \\
csDMARD (\%) & $88 \%$ & $90 \%$ & $94 \%$ & 0.973 \\
bDMARD (\%) & $22 \%$ & $81 \%$ & $59 \%$ & 0.003 \\
Mean prednisone dose (mg die) & $20.3 \pm 18.1$ & $15.8 \pm 14.4$ & $27.3 \pm 14.4$ & 0.252 \\
Concomitant csDMARD therapy (\%) & $71 \%$ & $71 \%$ & $59 \%$ & 0.865 \\
\hline
\end{tabular}

Table 2. Causes of treatment discontinuation of biologic agents in adult onset Still's Disease. TNFi = Tumor Necrosis Factor inhibitors

\begin{tabular}{lcccc}
\hline & $\begin{array}{c}\text { Anakinra } \\
(\mathbf{n}=\mathbf{4 1 )}\end{array}$ & $\begin{array}{c}\text { Tocilizumab } \\
(\mathbf{n}=\mathbf{2 1})\end{array}$ & TNFi (n=17) & Total $(\mathbf{n}=\mathbf{7 9})$ \\
\hline Inefficacy & $24 \%$ & $14 \%$ & $65 \%$ & $30 \%$ \\
Adverse events & $10 \%$ & $10 \%$ & $24 \%$ & $13 \%$ \\
Other reasons & $2 \%$ & $5 \%$ & $0 \%$ & $3 \%$ \\
Total & $37 \%$ & $29 \%$ & $88 \%$ & $46 \%$ \\
\hline
\end{tabular}

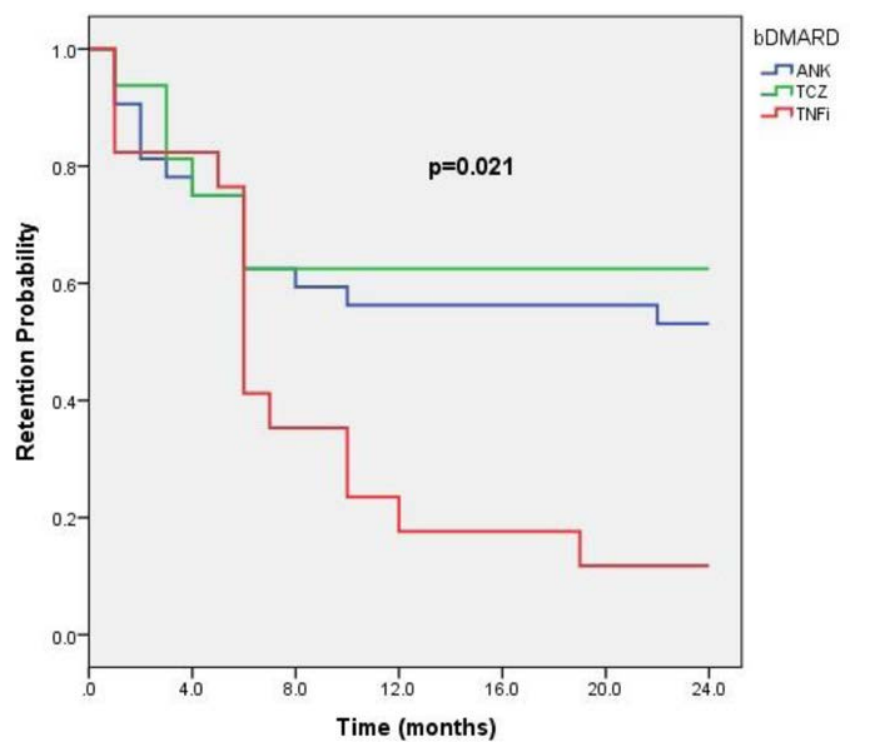

Figure 1. Kaplan-Meier curves comparing anakinra (ANK), tocilizumab (TCZ) and Tumo Necrosis Factor inhibitors (TNFi) at 24 months in adult onset Still's disease. 
Conclusion: In our AOSD cohort, DRRs at 24 months of TCZ and ANK were not statistically different, and they were both significantly higher than the DRR of TNFi. Previous use of biologic agents was found to have no effect on bDMARDs retention probability.

References:

[1] Efficacy and safety of biological agents in adult-onset Still's disease; Cavalli et al; Scand J Rheumatol. 2015;44(4):309-14.

Disclosure of Interests: Nicola Farina: None declared, Alessandro Tomelleri: None declared, Corrado Campochiaro Speakers bureau: Novartis, Pfizer, Roche, GSK, SOBI, Giacomo De Luca Speakers bureau: SOBI, Novartis, Celgene, Pfizer, MSD, Giulio Cavalli Speakers bureau: SOBI, Novartis, Pfizer, Elena Baldissera Speakers bureau: Novartis, Pfizer, Roche, Alpha Sigma, Sanofi, Lorenzo Dagna Grant/research support from: Abbvie, BMS, Celgene, Janssen, MSD, Mundipharma Pharmaceuticals, Novartis, Pfizer, Roche, SG, SOBI, Consultant of: Abbvie, Amgen, Biogen, BMS, Celltrion, Novartis, Pfizer, Roche, SG, and SOBI DOI: 10.1136/annrheumdis-2020-eular.3129

\section{SAT0520 \\ CONTROVERSY ON DIAGNOSIS AND TREATMENT OF ADULT PATIENTS WITH SAPHO SYNDROME: MULTI- DISCIPLINARY INTERNATIONAL SURVEY}

V. Furer $^{1,2}$, M. Kishimoto ${ }^{3}$, S. Tsuji ${ }^{4}$, Y. Taniguchi ${ }^{5}$, Y. Ishihara ${ }^{6}$, T. Tomita $^{7}$, O. Elkayam ${ }^{1,2} .{ }^{1}$ Tel Aviv Sourasky Medical Center, Tel Aviv-Yafo, Israel; ${ }^{2} T e l$ Aviv University, Sackler Faculty of Medicine, Tel Aviv-Yafo, Israel; ${ }^{3}$ Kyorin University School of Medicine, Department of Nephrology and Rheumatology, Mitaka, Japan; ${ }^{4}$ Osaka Minami Medical Center, Department of Rheumatology and Orthopaedic Surgery, Osaka, Japan; ${ }^{5}$ Kochi Medical School Hospital, Department of Endocrinology, Metabolism, Nephrology and Rheumatology, Nankoku, Japan; ${ }^{6}$ Japan Medical Research Foundation, Tokyo, Japan; ${ }^{7}$ Graduate School of Medicine and Faculty of Medicine, Osaka University, 6Department of Orthopaedic Biomaterial Science, Suita, Japan

Background: Synovitis, acne, pustulosis, hyperostosis, osteitis (SAPHO) is a rare disease involving skin and skeleton, with a potentially complicated and severe course, optimal management of which seems to require a collaborative rheumatology and dermatology care. Diagnostic criteria for SAPHO remain preliminary and lack validation. There are no evidence-based treatment algorithms in SAPHO due to lack of clinical trials in this rare medical condition.

Objectives: This study aimed to investigate the current practice in the diagnosis and treatment of SAPHO syndrome among the international rheumatology and dermatology communities.

Methods: We conducted a survey among the members of the Group for Research and Assessment of Psoriasis and Psoriatic Arthritis (GRAPPA) combining international rheumatologists and dermatologists as well as members of the Japanese and Israeli Societies of Rheumatology.

Results: A total of 78 physicians participated in the survey: rheumatologists $(83 \%, n=65)$, dermatologists $(11.5 \%, n=9)$, and orthopedics $(3.8 \%, n=3)$. SAPHO was considered a subtype of spondylarthritis by $48.7 \%(n=38)$, a subtype of psoriatic arthritis by $19.2 \%(n=15)$, a separate entity by $25.6 \%(n=20)$, and a subtype of reactive arthritis by $6.4 \%(n=5)$. Palmoplantar pustulosis was the most prevalent cutaneous manifestation $(n=44,56.4 \%)$ and anterior chest pain - the most prevalent osteoarticular manifestation $(n=66,84.6 \%)$. The majority $(84.6 \%$, $n=66$ ) voted for the update of the present diagnostic criteria by Khan 1994. Magnetic resonance imaging was considered the preferred imaging modality for the diagnosis of SAPHO by $41 \%(n=32)$. Conduction of bone biopsy for diagnosis of non-infectious osteitis was supported only by $10.3 \%(n=8)$. Patient-reported outcomes were considered the most appropriate measure for the assessment of disease activity by $47.4 \%(n=37)$. The treatment approach was overall similar among the rheumatology and dermatology communities, including non-steroidal anti-inflammatory drugs, bisphosphonates, conventional disease-modifying anti-inflammatory drugs, and biologics (Table 1).

\section{Table 1. Preferences in the treatment choice of SAPHO}

\begin{tabular}{lc}
\hline Treatment & \% of responders \\
\hline NSAIDs & $76.6 \%$ \\
Glucocorticoids & $32.5 \%$ \\
Conventional DMARDs & $57.1 \%$ \\
Bisphosphonates & $48.1 \%$ \\
Anti-TNF biologic therapy & $75.3 \%$ \\
Other biologic therapy & $20.8 \%$ \\
Antibiotic & $14.3 \%$ \\
Tonsillectomy & $5.1 \%$ \\
Isotretinoin & $5.2 \%$ \\
Topical therapy & $10.4 \%$ \\
Intra-articular steroid injection & $7.8 \%$
\end{tabular}

Legend: NSAIDs - non-steroidal anti-inflammatory drugs; DMARDs - disease modifying anti-rheumatic drugs
Conclusion: Our study underlines the controversy on diagnosis and treatment of SAPHO syndrome among specialists in rheumatology and dermatology and emphasizes an unmet need for update and validation of diagnostic criteria and treatment approach.

Acknowledgments: GRAPPA, Japanese Society of Rheumatology, Israeli Society of Rheumatology

Disclosure of Interests: Victoria Furer: None declared, Mitsumasa Kishimoto: None declared, Shigeyoshi Tsuji Grant/research support from: Eli Lilly, Speak ers bureau: AbbVie, Asahi Kasei, Chugai, Daiichi Sankyo, Eli Lilly, Eisai, Mitsubishi Tanabe, Celgene, and Novartis Pharma K.K., Yoshinori Taniguchi: None declared, Yoko Ishihara: None declared, Tetsuya Tomita Consultant of: Eli Lilly and Company, Ori Elkayam Speakers bureau: AbbVie, BMS, Pfizer, Roche, Sanofi-Aventis, Novartis, Jansen

DOI: 10.1136/annrheumdis-2020-eular.6390

\section{SAT0521}

\section{EVALUATION OF LIPID PROFILE IN PATIENTS WITH} AVASCULAR NECROSIS

K. Gudkevich ${ }^{1}$, N. Martusevich ${ }^{2}$, E. Dashkevich ${ }^{3} .{ }^{1}$ Minsk City Clinical Hospital No. 6, Rheomatology, Minsk, Belarus; ${ }^{2}$ Belarusian State Medical University, Minsk, Belarus; ${ }^{3}$ Minsk City Clinical Hospital No. 10, Minsk, Belarus

Background: Avascular necrosis (AN) is a disabling disease affecting predominantly young men. It steadily progresses to secondary osteoarthritis. The rate of disability is high, because hip joint is most commonly affected by AN; moreover the rate of bilateral hip joint involvement is also high. The key event in the pathogenesis of $\mathrm{AN}$ is circulation disorder which results in ischemia and, furthermore, death of bone tissue.

Nowadays the data about lipid metabolism in patients with AN. Besides, there is contradictory data about the role of homocysteine in the risk of cardiovascular events. Based on these, it is topical to investigate lipid metabolism and endothelial function in patients with AN.

Objectives: The aim of the present study was to investigate lipid metabolism and endothelial function in patients with different stages of AN in order to find risk factors for incidence and progression of this disease.

Methods: We recruited 41 patients with AN of the femoral head or femoral condyles aged $51(35-64)$ years, $56,1 \%(n=23)$ were male. The inclusion criteria was diagnosis of AN confirmed by X-Ray, CT or MRI. The exclusion criteria were: traumatic AN, Legg-Calve-Pertes disease, connective tissue systemic diseases. Also we divided patients into subgroups with early $(39 \%(n=16))$ and late $(61,0 \%$ $(n=25))$ stages of $A N$.

Patients of the control group $(n=32)$ were comparable by age and gender. The data was proceeded using non-parametric and descriptive statistics. We evaluated the following parameters: homocysteine, total cholesterol, high-density lipoprotein cholesterol, low-density lipoprotein cholesterol, high-density lipoprotein cholesterol, very low-density lipoprotein cholesterol, cholesterol ratio, apolipoprotein A1, apolipoprotein B.

Results: In patients with AN we observed higher level of homocysteine $(12,119$ $(7,771-14,38)$ vs. $7,911(6,612-10,54) \mathrm{mcmol} / \mathrm{l}, \mathrm{p}=0,05)$. The results are consistent with the data that higher homocyseine level is associated with higher risk for cardiovascular events (1). There is literature data about elevated homocysteine level in patients with AN, which was associated with lower levels of vitamin B12 and folic acid. Thus, interpretation of homocystene significance is more reliable in association with vitamin B12 and folic acid levels.

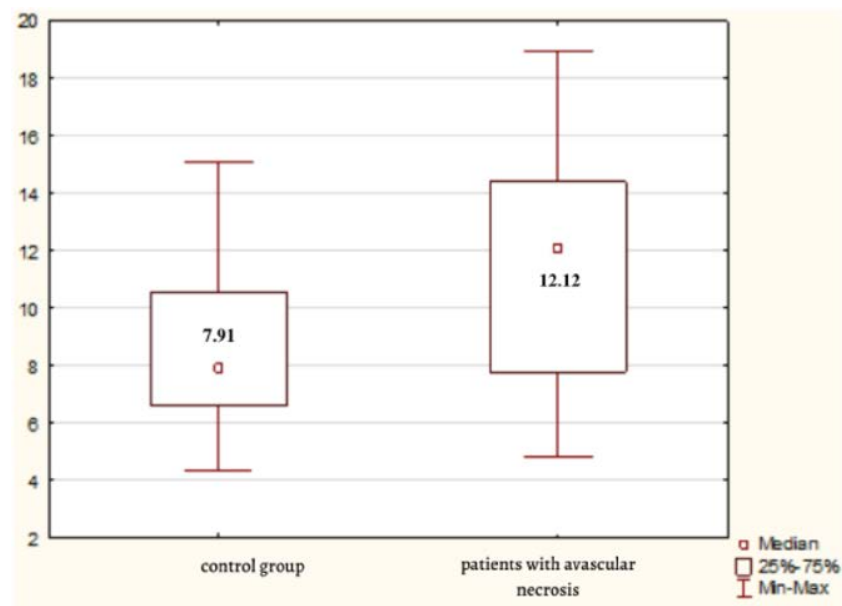

Figure 1. Homocysteine levels in patients of control group and in patients with avascular necrosis 